\title{
AIP
}

\section{An integrated micro-chip for rapid detection of magnetic particles}

Chinthaka P. Gooneratne, Cai Liang, Ioanna Giouroudi, and Jürgen Kosel

Citation: Journal of Applied Physics 111, 07B327 (2012); doi: 10.1063/1.3678303

View online: http://dx.doi.org/10.1063/1.3678303

View Table of Contents: http://scitation.aip.org/content/aip/journal/jap/111/7? ver=pdfcov

Published by the AIP Publishing

\section{Articles you may be interested in}

Quantitative detection of magnetic particles in a chromatographic membrane by a giant magnetoresistance sensor

J. Appl. Phys. 105, 07E713 (2009); 10.1063/1.3075868

Optimization of the magnetic noise and sensitivity of giant magnetoimpedance sensors

J. Appl. Phys. 103, 033903 (2008); 10.1063/1.2837106

On-chip separation of magnetic particles with different magnetophoretic mobilities

J. Appl. Phys. 101, 024913 (2007); 10.1063/1.2423137

Detection of ferromagnetic particles using spin valve sensors

J. Appl. Phys. 100, 044909 (2006); 10.1063/1.2234537

Giant magnetoresistance monitoring of magnetic picodroplets in an integrated microfluidic system

Appl. Phys. Lett. 85, 4783 (2004); 10.1063/1.1825059

MIT LINCOLN

LABORATORY CAREERS

Discover the satisfaction of innovation and service

to the nation
- Space Control

- Air \& Missile Defense

- Communications Systems \& Cyber Security

- Intelligence, Surveillance and

Reconnaissance Systems

- Advanced
Electronics
- Tactical Systems
- Homeland
Protection
- Air Traffic Control

LINCOLN LABORATORY

MassachusetTs Institute of TeChNOLOgY

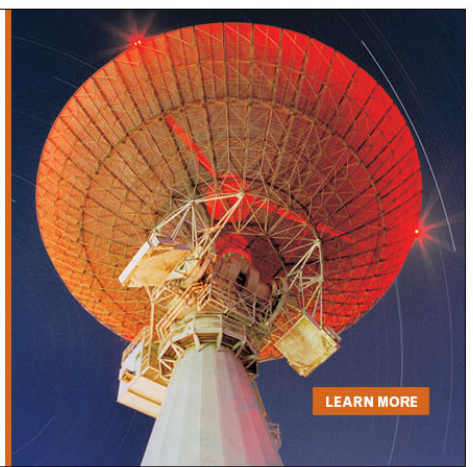




\title{
An integrated micro-chip for rapid detection of magnetic particles
}

\author{
Chinthaka P. Gooneratne, ${ }^{1, \text { a) }}$ Cai Liang, ${ }^{1}$ Ioanna Giouroudi, ${ }^{2}$ and Jürgen Kosel ${ }^{1}$ \\ ${ }^{1}$ Division of Physical Sciences and Engineering, King Abdullah University of Science and Technology, \\ Thuwal 23955, Kingdom of Saudi Arabia \\ ${ }^{2}$ Institute of Sensor and Actuator Systems, Vienna University of Technology, 1040 Vienna, Austria
}

(Presented 3 November 2011; received 23 September 2011; accepted 28 November 2011; published online 9 March 2012)

This paper proposes an integrated micro-chip for the manipulation and detection of magnetic particles (MPs). A conducting ring structure is used to manipulate MPs toward giant magnetoresistance (GMR) sensing elements for rapid detection. The GMR sensor is fabricated in a horseshoe shape in order to detect the majority of MPs that are trapped around the conducting structure. The GMR sensing elements are connected in a Wheatstone bridge circuit topology for optimum noise suppression. Full fabrication details of the micro-chip, characterization of the GMR sensors, and experimental results with MPs are presented in this paper. Experimental results showed that the micro-chip can detect MPs from low concentration samples after they were guided toward the GMR sensors by applying current to the conducting ring structure. (C) 2012 American Institute of Physics. [doi:10.1063/1.3678303]

Giant magnetoresistance (GMR) sensor based microchips for biological or medical applications have taken advantage of the fact that magnetic particles (MPs) can be transported to a sensing location by the application of magnetic forces. ${ }^{1}$ Research on utilizing high gradient current lines to guide magnetically labeled MPs to the sensing area, probe immobilization over the sensing area to capture and identify target bio-samples and detection by sensitive GMR sensors is well documented. ${ }^{2-9}$ Highly sensitive GMR sensor-based micro-chips have been able to actuate and detect a single $\mathrm{MP},{ }^{10}$ detect oligonucleotides hybridization events in the femtomolar range, ${ }^{11}$ and detect sub-picomolar concentrations of proteins. ${ }^{12}$

In this research, first a micro-trap consisting of conducting rings is designed to precisely transport a small number of MPs in a controlled manner over long distances by magnetic forces enabling the rapid concentration of a majority of target cells to a sensing site for detection. This is especially important in low concentration bio-samples. ${ }^{13-15}$ The number of rings in the conducting structure can be customized according to the size of the area, from which the MPs need to be attracted, trapped, and transported without the need to increase the number of switches or to unduly increase the complexity of the fabrication. By grouping the rings into sets the same number of switches can be used. Unlike square, meander or mesh structures, which have high gradients at the corners, a circular microstructure has a uniform magnetic field and force along the periphery of the ring. Therefore, the surface coverage of the MPs will be homogenous around the circular element. By switching current to different rings, MPs can be manipulated and immobilized on the innermost ring where the GMR sensors are located. Second, for optimum measurement capability with high spatial resolution, horseshoe shaped spin valve type GMR sensors are fabricated directly underneath and all along the innermost ring to

\footnotetext{
a) Author to whom correspondence should be addressed. Electronic mail: chinthaka.gooneratne@kaust.edu.sa.
}

detect the fringing fields originating from the MPs. By means of, e.g., probe immobilization, the proposed structure could be employed to detect biological targets.

MPs are guided to the sensing zone by the conducting micro-ring structure shown in Fig. 1(a). The location of force maxima can be controlled by switching current to different micro-rings. ${ }^{16}$ Hence, MPs can be guided from the outer micro-ring to the innermost ring where the GMR sensors are located, directly underneath and separated from the ring structure by a thin passivation layer. This procedure can be repeated several times to ensure a high concentration rate of MPs at the sensing zone.

The micro-chip has four GMR sensing elements with two active and two reference sensors in a Wheatstone bridge architecture as shown in Figs. 1(b) and 1(c). The magnetic field produced by the innermost micro-ring is in the sensitive plane of the active GMR sensors producing a considerable resistance change, which has to be taken into consideration to ensure high sensitivity. To address this issue, a conducting micro-ring is also located above the reference sensors. Under the influence of an external magnetic field MPs produce fringing magnetic fields that affect only GMRs 1 and 2, changing their resistance values in opposite directions. This produces a measurable differential voltage output.

The spin valves were deposited using a magnetron sputtering system on a oxide coated Silicon wafer with the following "bottom pinned" structure: Substrate/MgO 15/ $\mathrm{Ni}_{80} \mathrm{Fe}_{20} 2.5 / \mathrm{Ir}_{17} \mathrm{Mn}_{83}{ }_{9} / \mathrm{Co}_{50} \mathrm{Fe}_{50}$ 4.5/Ru $0.8 / \mathrm{Co}_{50} \mathrm{Fe}_{50} \quad 1.0 /$ $\mathrm{Ni}_{80} \mathrm{Fe}_{20} 4 / \mathrm{Co}_{50} \mathrm{Fe}_{50} 1.5 / \mathrm{Cu} 4 / \mathrm{Co}_{50} \mathrm{Fe}_{50} 0.8 / \mathrm{Ni}_{80} \mathrm{Fe}_{20} 5 / \mathrm{Ru} 1$ (all thicknesses in nanometers). A thickness of $0.8 \mathrm{~nm}$ was chosen for $\mathrm{Ru}$ as it provides a very strong antiparallel coupling between CoFe layers. After deposition spin valve elements were patterned to a horseshoe shape by e-beam lithography followed by ion beam milling. To achieve a linear sensor output a transverse magnetization direction (with respect to the horseshoe sensor arc line) was induced in the pinned layer during annealing at a temperature of $280^{\circ} \mathrm{C}$ under an applied magnetic field of $220 \mathrm{mT}$, whereas the free 


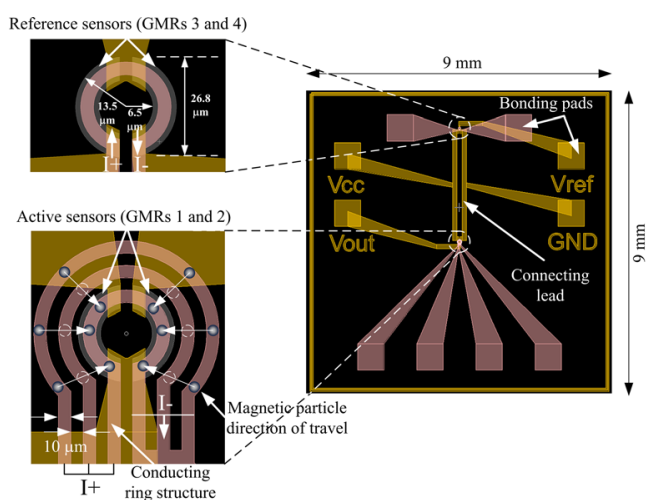

(a)

(b)

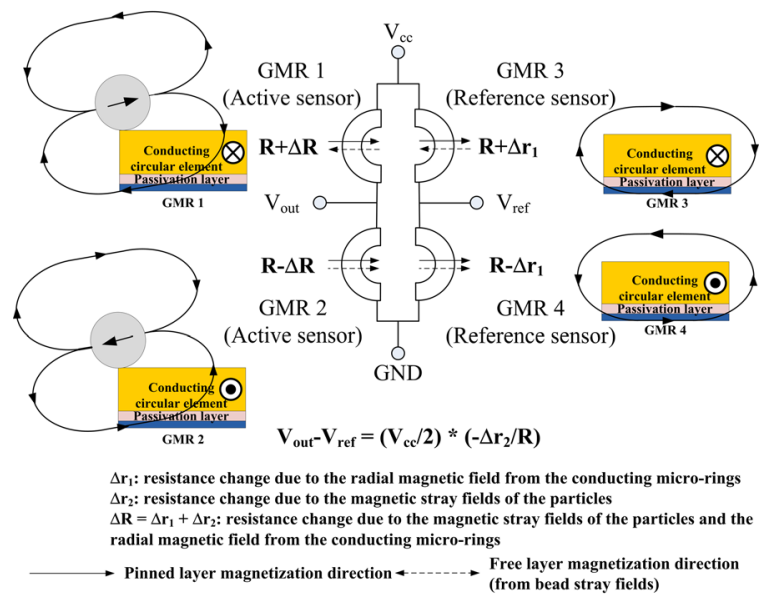

(c)

FIG. 1. (Color online) Schematic layout of the GMR micro-chip. (a) Active and reference sensing areas, (b) chip layout, and (c) Wheatstone bridge connection of GMR sensors.

layer was free to rotate around the longitudinal direction (horseshoe sensor arc line). The conducting micro-ring structure was fabricated of $500 \mathrm{~nm}$ thick Au with a $10 \mathrm{~nm}$ thick $\mathrm{Ti}$ adhesion layer on top of the sensor using photolithography and lift-off techniques. The fabricated micro-chip is shown in Fig. 2.

The resistance of the GMR sensing element was measured to be $338 \Omega$. The maximum magnetoresistance is approximately $3.6 \%$ and the sensitivity in the linear region is approximately $0.5 \% / \mathrm{mT}$ when a DC magnetic field is applied in parallel to the pinned direction, for a bias current of $5 \mathrm{~mA}$ (Fig. 3(a)). Large spacer thicknesses lead to enhanced current shunting and scattering effects. Fig. 3(b) shows that the magnetoresistance curve is almost symmetrical when a DC magnetic field is applied transverse to the pinned direction since the transverse component to the applied field generates a symmetric component and the asymmetric response can only be generated by the parallel component. ${ }^{17}$ The small signal AC sensitivity of the GMR sensing element in its sensitive axis (parallel to the pinned direction) is approximately $0.3 \mathrm{mV} / \mathrm{mT}$ as shown in Fig. 3(c) for a magnetic field of approximately $0.48 \mathrm{mT}$ at a frequency of $10 \mathrm{~Hz}$.

The micro-chip was placed under a microscope equipped with a CCD camera. The experimental process involved adding $2 \mu \mathrm{l}$ of $2 \mu \mathrm{m}$ MPs from Micromod® with a diluted concentration of $1 \mathrm{mg} / \mathrm{ml}$ by a pipette to the area where the active GMR sensors are located. A circular microscope glass slide was

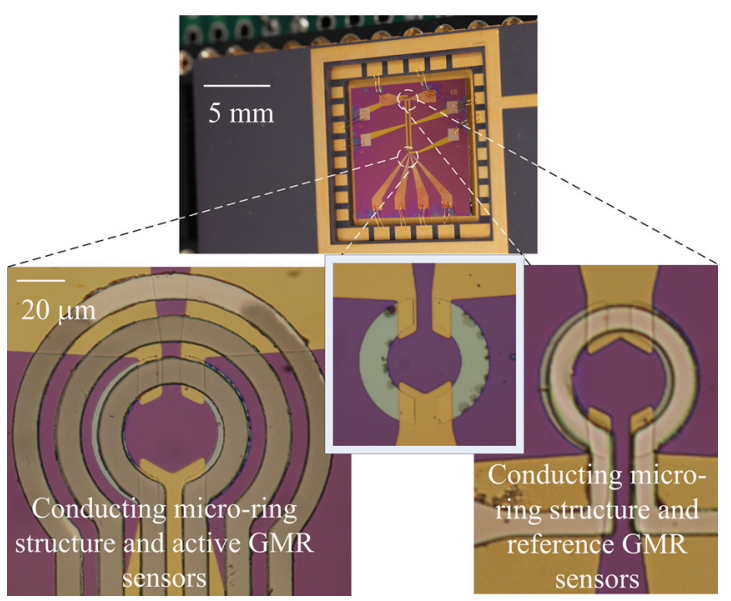

FIG. 2. (Color online) Fabricated GMR micro-chip (inset: fabricated horseshoe sensing elements that are underneath the innermost conducting micro-ring).

placed on top of the liquid in order to obtain a clear image with the microscope and to confine the MPs to the active sensing area. A stable DC bias current of $5 \mathrm{~mA}$ was applied to the GMR bridge circuit by means of a current source meter. An AC current of $20 \mathrm{~mA}$ at a frequency of $10 \mathrm{~Hz}$ was applied to the outermost micro-ring by a function generator. The current was switched to the middle ring after $18 \mathrm{~s}$ and finally to the innermost ring after a further $18 \mathrm{~s}$. Once the MPs were directly on top of the GMR sensors the bridge output was observed utilizing a lock-in amplifier for $40 \mathrm{~s}$ before finally flushing the MP solution from the micro-chip surface using a pipette. Figure 4(a) shows how the MPs are randomly scattered when initially injected onto the micro-chip. Also, the few MPs trapped at the active GMR sensors are due to the fact that the bias current of the GMR sensor produces small magnetic stray fields that apply a force on nearby MPs. However, the GMR sensors normally need to be activated only after the MPs are trapped at the innermost ring and not from the beginning of the experiment. It can be seen from Fig. 4(b) that at the conclusion of the manipulation procedure MPs are concentrated and trapped around the GMR sensing area. Large surface coverage of the sensing element ensures an accurate and high-resolution output signal.

When the MP solution is first injected to the micro-chip, no current is applied to the conducting micro-rings or GMR sensor and the output signal is zero (Fig. 4(c)). A signal output was observed once current was applied to the GMR sensors and the outermost conducting micro-ring due to the MPs that were attracted to the magnetic field produced by the GMR bias current. When current is switched from the outer ring to the middle ring, the MPs trapped at the outer ring move to the middle ring. In addition, some of the MPs trapped at the GMR sensor by the bias current through the GMR sensor move to the middle ring, which can be observed as a decrease in the output signal. Once current was applied to the innermost ring the majority of the magnetic MPs were concentrated on top of the GMR sensing elements as shown in Fig. 4(b) resulting in a significant increase in the output signal as shown in Fig. 4(c). The signal then decreased rapidly when the current to the micro-ring was turned off and the MPs were washed away by applying DI water but did not reduce to zero. The current to the inner micro-ring was 


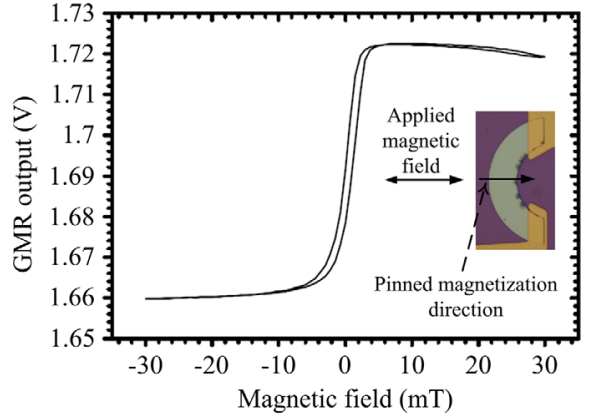

(a)

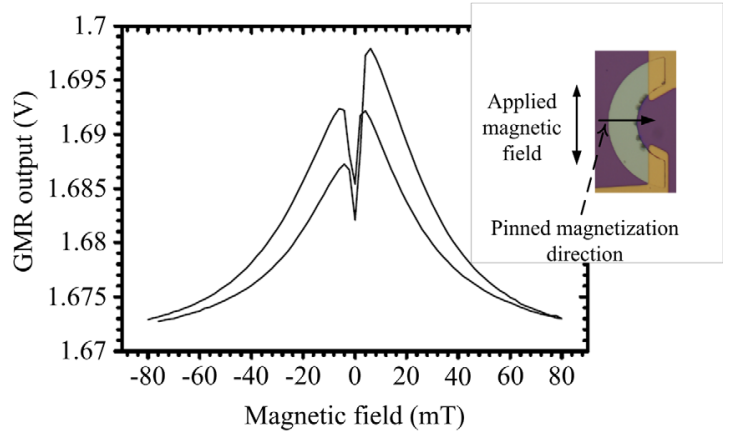

(b)

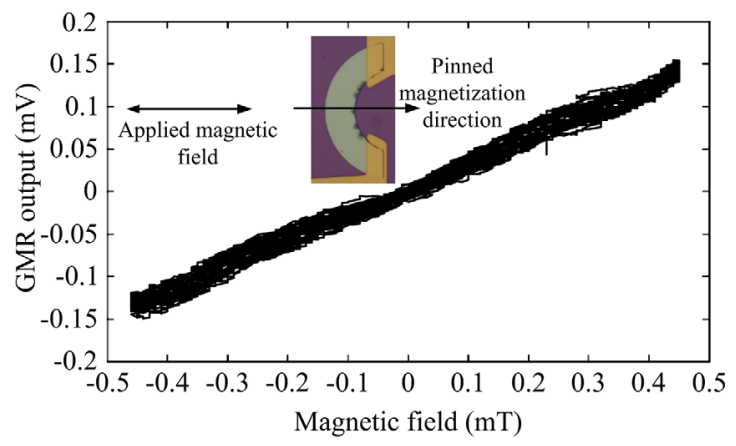

(c)

FIG. 3. (Color online) Characterization results of a single GMR sensing element. (a) DC characteristics (applied field is parallel to the pinned direction), (b) DC characteristics (applied field is transverse to the pinned direction), and (c) small signal AC characteristics.

turned on again to make sure the signal change was in fact due to the attracted MPs. The non-zero output signal can be attributed to the fact that there were few MPs still attached to the GMR sensor which can be solved by incorporating the micro-chip with a microfluidic setup and thoroughly washing in a microfluidic channel.

An integrated micro-chip for MP concentration and detection using conducting micro-structures and GMR sensors was reported. Experiments performed with the integrated micro-chip showed that it was able to combine both manipulation and detection of MPs. This method does not require a magnetic field produced by an external magnet and can reduce the measurement time and/or increase the measurement sensitivity. The surface area of the sensor was not optimally utilized due to the MPs attracted and trapped at the edge of the conductors. A possible improvement would be to displace the sensor so that its arc is below the edge of the conductor. Moreover, in this case a magnetic field nearly perpendicular to the plane can be used to magnetize the MPs, which ideally
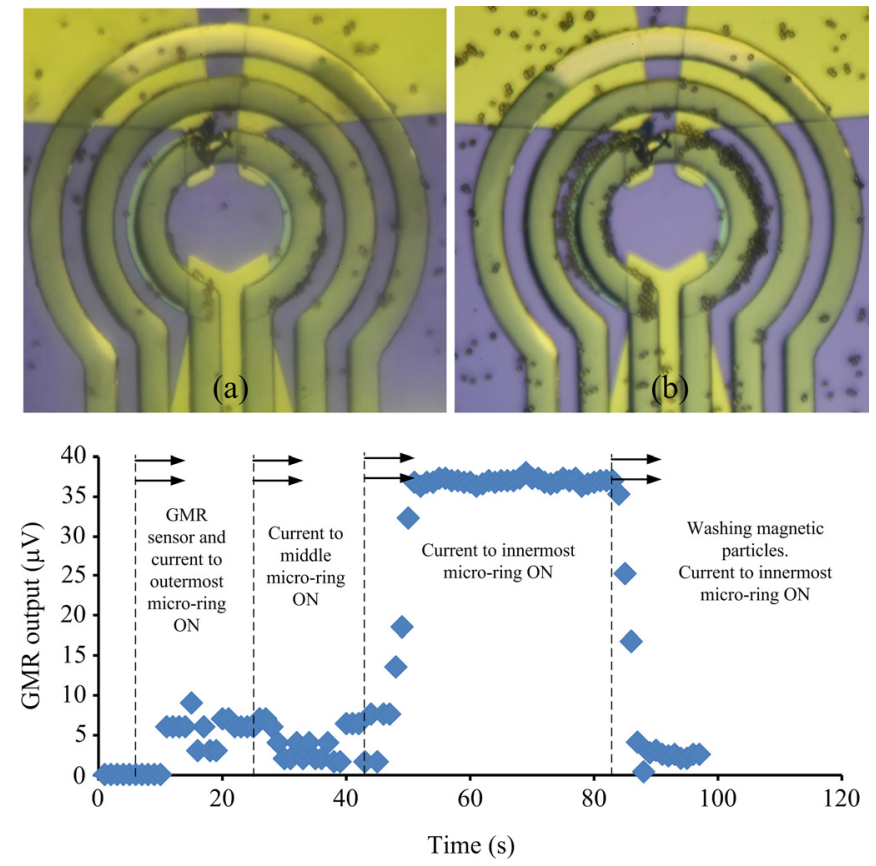

(c)

FIG. 4. (Color online) Experimental results showing manipulation of magnetic particles towards the sensing area. (a) No current applied. (b) Current applied to innermost micro-ring concentrating magnetic particles to the active GMR sensors. (c) Experimental results showing the variation of GMR sensor output at different steps of the manipulation process.

does not strongly influence the sensor. Therefore, larger magnetic fields can be used and larger stray fields due to a higher magnetization of the magnetic particle can occur. The unique micro-chip explored in this paper has the potential to be integrated in a compact chip for biological or medical analysis.

The authors gratefully acknowledge Dr. Volker Hoeink and Dr. Hubert Brückl of the Austrian Institute of Technology for their help in GMR sensor fabrication. The authors also thank the staff at the KAUST microfabrication/nanofabrication laboratory, especially Dr. Ren Jian, Dr. Zhihong Wang, Mr. Xiang Yu, Mr. Basil Chew, and Mr. Kim Chong Wong, and also Mr. Guodong Li from the electronic workshop, for their help in microfabrication and electronic circuitry, respectively.

${ }^{1}$ C. Liu et al., J. Appl. Phy. 105, 102014 (2009).

${ }^{2}$ M. Megens and M. Prins, J. Magn. Magn. Mater. 293, 702 (2005).

${ }^{3}$ P. P. Freitas et al., in Magnetoelectronics, edited by M. Johnson (Academic, New York, 2004).

${ }^{4}$ D. L. Graham et al., J. Appl. Phys. 91, 7786 (2002).

${ }^{5}$ L. Lagae et al., J. Appl. Phys. 91, 7445 (2002).

${ }^{6}$ D. L. Graham et al., Sens. Act B. 107, 936 (2005).

${ }^{7}$ R. Wirix-Speetjens et al., Sens. Act B. 128, 1 (2007).

${ }^{8}$ B. M. de Boer et al., Biosens. Bioelec. 22, 2366 (2007).

${ }^{9}$ C. R. Tamanaha et al., Biosens. Bioelec. 24, 1 (2008).

${ }^{10}$ R. C. Chavez et al., J. Appl. Phys. 109, 064702 (2011).

${ }^{11}$ V. C. Martins et al., Biosens. Bioelec. 24, 2690 (2009).

${ }^{12}$ W. U. Dittmer et al., J. Immunol. Methods 338, 40 (2008).

${ }^{13}$ J. Chalmers et al., Biotechnol. Bioeng. 64, 519 (1999).

${ }^{14} \mathrm{R}$. Powles et al., Lancet 355, 1231 (2000).

${ }^{15}$ O. Lara et al., Exp. Hematol. 32, 891 (2004).

${ }^{16}$ C. P. Gooneratne et al., J. Appl. Phys. 109, 07E517 (2011).

${ }^{17}$ S. Mao et al., J. Appl. Phys. 85, 5033 (1999). 